\title{
An Adaptive Unscented Kalman Filtering Algorithm for MEMS/GPS Integrated Navigation Systems
}

\author{
Jianhua Cheng, ${ }^{1,2}$ Daidai Chen, ${ }^{1}$ Rene Jr. Landry, ${ }^{2}$ Lin Zhao, ${ }^{1}$ and Dongxue Guan' \\ ${ }^{1}$ Marine Navigation Research Institute, College of Automation, Harbin Engineering University, Harbin 150001, China \\ ${ }^{2}$ LASSENA Laboratoire, Ecole de Technologie Superieure, Université du Québec, Montréal, Canada H3C 1K3 \\ Correspondence should be addressed to Daidai Chen; ins_dai@163.com
}

Received 13 November 2013; Accepted 15 February 2014; Published 20 March 2014

Academic Editor: Yuxin Zhao

Copyright (c) 2014 Jianhua Cheng et al. This is an open access article distributed under the Creative Commons Attribution License, which permits unrestricted use, distribution, and reproduction in any medium, provided the original work is properly cited.

\begin{abstract}
MEMS/GPS integrated navigation system has been widely used for land-vehicle navigation. This system exhibits large errors because of its nonlinear model and uncertain noise statistic characteristics. Based on the principles of the adaptive Kalman filtering (AKF) and unscented Kalman filtering (AUKF) algorithms, an adaptive unscented Kalman filtering (AUKF) algorithm is proposed. By using noise statistic estimator, the uncertain noise characteristics could be online estimated to adaptively compensate the timevarying noise characteristics. Employing the adaptive filtering principle into UKF, the nonlinearity of system can be restrained. Simulations are conducted for MEMS/GPS integrated navigation system. The results show that the performance of estimation is improved by the AUKF approach compared with both conventional AKF and UKF.
\end{abstract}

\section{Introduction}

Microelectromechanical systems (MEMS) and Global Positioning System (GPS) integrated navigation system have the advantages of small size, light weight, and low cost, but, because of its low accuracy, it can only be applied in low accuracy navigation fields such as unmanned aircrafts and land-vehicles [1,2]. There are three main factors impacting its performance: (a) the inaccuracy of model parameters, (b) the uncertainty of measurement and observation noise statistic properties, and (c) the nonlinearity of model $[3,4]$.

The classical Kalman filter (KF) provides a recursive solution for estimation of linear dynamic systems. The optimality of the KF algorithm is mainly dependent on a priori statistic of the process and measurement noise and the linear system model. However, if the priori information is insufficient or biased, the precision of the estimated states will be degraded, even leading to divergences [5]. In the case of MEMS/GPS applications, the estimation system tends to be nonlinear as well as variational noise properties [6]. Meanwhile, for the vehicle navigation, there are many sudden motion changes. To deal with these problems, the implement of AKF appears to be one of suitable approaches [7].
By utilizing the innovation and residual information, the AKF could adapt the filter stochastic properties online to accommodate itself to changes in vehicle dynamics. Thus, this technique could reduce the reliance of filter on the prior statistical information and obtain the noise statistic parameters of the dynamic system. The essence of AKF is to adapt the filter weights, so as to restrain model errors and improve the accuracy of filters. It is showed that applying AKF to the INS/GPS integrated navigation system could obtain better estimated performance than by using conventional KF, especially less than $20 \%$ root mean square error in attitude estimation [8]. However, AKF is unreliable when it is applied into the nonlinear applications.

UKF, which is another extension of Kalman filter, could give reliable estimates even if the nonlinearities of system are quiet severe [9]. The linearization procedure is avoided by introducing the unscented transformation (UT), which is a method to approximate the joint distribution of states and measurements variables. In UT, a number of sigma points are chosen which could maintain the desired mean and covariance of states. Theoretically, the performance of UKF could be close to that of the three-order Taylor series expansion approximation or better than it [10]. However, 
many kinds of systems such as aircrafts, ships, and spacecrafts would be intensively disturbed by external environment. As a result, the statistical properties for system process and measurement noises cannot be predicted, and UKF cannot solve these problems effectively.

As a combination of AKF and UKF, the adaptive UKF has been developed and applied to nonlinear joint estimation of both time-varying states and parameters [11]. The adaptive principles have been employed to update the means and covariances of the process and measurement noises online. The contributions of model predicted states and measurement information for filtering are balanced. In this paper, a new AUKF algorithm is proposed for MEMS/GPS integrated navigation systems in vehicle applications. A noise estimator for UKF is designed to estimate and update the means and covariances of noises online. Then, the updated means and covariances are propagating through the UKF. The proposed AUKF has the adaptive ability to time-varying noises and the noise estimates are unbiased. The performance of AUKF applied in land navigation is evaluated by simulations and the results show that the integrated system exhibits excellent robustness and navigation performance.

\section{Unscented Kalman Filtering Algorithm for Nonzero Mean Noise}

In the integrated navigation field, almost all of the systems are nonlinear. The general nonlinear discrete system model is given as

$$
\begin{gathered}
x_{k}=f_{k-1}\left(x_{k-1}\right)+w_{k-1}, \\
z_{k}=h_{k}\left(x_{k}\right)+v_{k},
\end{gathered}
$$

where $x_{k} \in R^{n}$ is the state vector, $z_{k} \in R^{m}$ is the measurement vector, and $f_{k}(\cdot) \in R^{n \times n}$ and $h_{k}(\cdot) \in R^{m \times n}$ are the state and measurement matrices of nonlinear system, respectively. $w_{k}$ and $v_{k}$ are the Gaussian white noise which are unrelated. The mean and covariance of $w_{k}$ and $v_{k}$ are given as follows:

$$
\begin{array}{rlrl}
E\left[w_{k}\right] & =q, & \operatorname{cov}\left[w_{k} w_{j}^{T}\right]=Q \delta_{k j} ; \\
E\left[v_{k}\right]=r, & \operatorname{cov}\left[v_{k} v_{j}^{T}\right]=R \delta_{k j},
\end{array}
$$

where $q$ and $r$ are nonzero constant variables and $\delta_{k j}$ is a Kronecker delta function.

The initial state $x_{0}$ is uncorrelated with both the process noise and measurement noise. These initial states exhibit Gaussian normal distributions. The prior mean and covariance matrices of $x_{0}$ are defined by

$$
\begin{gathered}
\widehat{x}_{0}=E\left(x_{0}\right), \\
P_{0}=\operatorname{cov}\left(x_{0}\right)=E\left(x_{0}-\widehat{x}_{0}\right)\left(x_{0}-\widehat{x}_{0}\right)^{T} .
\end{gathered}
$$

Assuming that $\mu_{k}=w_{k}-q$ and $\eta_{k}=v_{k}-r$ and substituting them into (1) yield

$$
\begin{gathered}
x_{k}=f_{k-1}\left(x_{k-1}\right)+q+\mu_{k-1}, \\
z_{k}=h_{k}\left(x_{k}\right)+r+\eta_{k},
\end{gathered}
$$

where the mean and covariance of $\mu_{k}$ and $\eta_{k}$ are given as follows:

$$
\begin{array}{ll}
E\left[\mu_{k}\right]=0, & \operatorname{cov}\left[\mu_{k} \mu_{j}^{T}\right]=Q \delta_{k j} ; \\
E\left[\eta_{k}\right]=0, & \operatorname{cov}\left[\eta_{k} \eta_{j}^{T}\right]=R \delta_{k j} .
\end{array}
$$

According to system model of (4), the recursive solution of UKF algorithm is noted as follows.

(1) Sigma Points Sampling. In order to guarantee positive semidefinite of state covariance, the modified sigma points sampling solution based on the scaling method is adopted [12].

Choose $2 n+1$ sigma points $\xi_{i, k / k-1}$ as follows:

$$
\begin{gathered}
\xi_{0, k / k-1}=\bar{x} \\
\xi_{i, k / k-1}=\bar{x}+(\alpha \sqrt{(n+\lambda) P})_{i}, \quad i=1,2, \ldots, n, \\
\xi_{i, k / k-1}=\bar{x}-(\alpha \sqrt{(n+\lambda) P})_{i-L}, \quad i=n+1, n+2, \ldots, 2 n,
\end{gathered}
$$

where $P$ is the covariance of the state vector $x, \alpha \sqrt{(n+\lambda) P}$ is the matrix square root of $n P$, and $(\alpha \sqrt{(n+\lambda) P})_{i}$ denotes the $i$ th row items of $\alpha \sqrt{(n+\lambda) P}$.

(2) Prediction. Propagating these sigma points $\xi_{i, k / k-1}$ through nonlinear state function $f_{k}(\cdot)+q$, we obtain [13]

$$
\gamma_{i, k / k-1}=f_{k-1}\left(\xi_{i, k / k-1}\right)+q, \quad i=0,1, \ldots, 2 n .
$$

Then, computing the predicted state $\widehat{x}_{k / k-1}$, the predicted covariance $P_{k / k-1}$ is as follows:

$$
\begin{gathered}
\widehat{x}_{k / k-1}=\sum_{i=0}^{L} W_{i}^{m} \gamma_{i, k / k-1}=\sum_{i=0}^{L} W_{i}^{m} f_{k-1}\left(\xi_{i, k / k-1}\right)+q \\
P_{k / k-1}=\sum_{i=0}^{L} W_{i}^{c}\left(\gamma_{i, k / k-1}-\widehat{x}_{k / k-1}\right)\left(\gamma_{i, k / k-1}-\widehat{x}_{k / k-1}\right)^{T}+Q
\end{gathered}
$$

where $W_{i}^{m}$ and $W_{i}^{c}$ are associated weights:

$$
\begin{aligned}
W_{0}^{m} & =\frac{\lambda}{(n+\lambda)}, \\
W_{0}^{c} & =\frac{\lambda}{(n+\lambda)}+\left(1-\alpha^{2}+\beta\right), \\
W_{i}^{m} & =\frac{1}{2(n+\lambda)}, \quad i=1,2, \ldots, 2 n, \\
W_{i}^{c} & =\frac{1}{2(n+\lambda)}, \quad i=1,2, \ldots, 2 n .
\end{aligned}
$$

Parameter $\lambda$ is a scaling parameter, which is defined by

$$
\lambda=\alpha^{2}(n+\kappa)-n
$$


The parameters $\alpha, \beta$, and $\kappa$ are the positive constants in the sampling method.

Then, propagating the sigma points $\xi_{i, k / k-1}$, the measurement function $h_{k}(\cdot)+r$ yields

$$
x_{i, k / k-1}=h_{k}\left(\xi_{i, k / k-1}\right)+r, \quad i=0,1, \ldots, 2 n .
$$

Computing the predicted measurement vector $\widehat{z}_{k / k-1}$, the covariance of the measurement $P_{\widetilde{z}_{k}}$ and the cross-covariance of the state and measurement $P_{\widetilde{x}_{k} \tilde{z}_{k}}$ are as follows:

$$
\begin{gathered}
\widehat{z}_{k / k-1}=\sum_{i=0}^{L} W_{i}^{m} x_{i, k / k-1}=\sum_{i=0}^{L} W_{i}^{m} h_{k}\left(\xi_{i, k / k-1}\right)+r, \\
P_{\widetilde{z}_{k}}=\sum_{i=0}^{L} W_{i}^{c}\left(x_{i, k / k-1}-\widehat{z}_{k / k-1}\right)\left(x_{i, k / k-1}-\widehat{z}_{k / k-1}\right)^{T}+R, \\
P_{\widetilde{x}_{k} \widetilde{z}_{k}}=\sum_{i=0}^{L} W_{i}^{c}\left(\gamma_{i, k / k-1}-\widehat{x}_{k / k-1}\right)\left(x_{i, k / k-1}-\widehat{z}_{k / k-1}\right)^{T} .
\end{gathered}
$$

(3) Updating. Then, computing the filter gain $K_{k}$, the updated state $\widehat{x}_{k / k}$ and covariance $P_{k / k}$ are as follows:

$$
\begin{gathered}
K_{k}=P_{\widetilde{x}_{k} \widetilde{z}_{k}}\left(P_{\widetilde{z}_{k}}\right)^{-1}, \\
\widehat{x}_{k / k}=\widehat{x}_{k / k-1}+K_{k}\left(z_{k}-\widehat{z}_{k / k-1}\right), \\
P_{k / k}=P_{k / k-1}-K_{k} P_{\widetilde{z}_{k}}\left(K_{k}\right)^{T} .
\end{gathered}
$$

\section{Noise Statistic Estimator}

Aiming at the uncertainty of process and measurement noise statistic properties, the measurement information are used to real time estimate and update the means and covariances of noises. Assume that $w_{k}$ and $v_{k}$ are uncorrelated and obey the Gaussian distributions. Based on the maximum a posterior (MAP) principle, a noise statistic estimator is derived.

The noise parameters $q, r$ and the noise matrices $Q$, $R$ are unknown and need to be estimated according to the updated measurements. The MAP estimates of $q, Q$, $r, R$ and the state $x_{k}$ are denoted as $\widehat{q}, \widehat{Q}, \widehat{r}, \widehat{R}$, and $\widehat{x}_{j / k}$, respectively. The conditional distribution of interest based on the measurements is expressed as

$$
J=p\left[X_{k}, q, Q, r, R \mid Z_{k}\right] .
$$

Because $p\left[Z_{k}\right]$ is disrelated to other parameters except the estimate state, the problem in calculating (16) changes to calculate the joint conditional probability distribution:

$$
\begin{aligned}
J & =p\left[X_{k}, q, Q, r, R, Z_{k}\right] \\
& =p\left[X_{k} \mid q, Q, r, R\right] p\left[Z_{k} \mid X_{k}, q, Q, r, R\right] p[q, Q, r, R]
\end{aligned}
$$

where $p[q, Q, r, R]$ can be treated as a constant which is provided by the prior statistic information.
The original problem has changed to calculate the conditional probability distributions $p\left[X_{k} \mid q, Q, r, R\right]$ and $p\left[Z_{k} \mid\right.$ $\left.X_{k}, q, Q, r, R\right]$.

According to the Gaussian distributions of $\mu_{k}$, the conditional probability distribution $p\left[X_{k} \mid q, Q, r, R\right]$ could be expressed as

$$
\begin{aligned}
p\left[X_{k} \mid q, Q, r, R\right] & p\left[x_{0}\right] \prod_{j=1}^{k} p\left[x_{j} \mid x_{j-1}, q, Q\right] \\
= & \frac{1}{(2 \pi)^{n / 2}\left|P_{0}\right|^{1 / 2}} \exp \left\{-\frac{1}{2}\left\|x_{0}-\widehat{x}_{0}\right\|_{P_{0}^{-1}}^{2}\right\} \\
& \times\left\{\prod_{j=1}^{k} \frac{1}{(2 \pi)^{n / 2}|Q|^{1 / 2}}\right. \\
= & C_{1}\left|P_{0}\right|^{-1 / 2}|Q|^{-k / 2} \\
& \left.\times \exp \left\{-\frac{1}{2}\left\|x_{0}-\widehat{x}_{0}\right\|_{P_{0}^{-1}}^{2}\left\|x_{j}-f_{j-1}\left(x_{j-1}\right)-q\right\|_{Q^{-1}}^{2}\right\}\right\} \\
& \left.+\sum_{j=1}^{k}\left\|x_{j}-f_{j-1}\left(x_{j-1}\right)-q\right\|_{Q^{-1}}^{2}\right\},
\end{aligned}
$$

where $n$ is the dimension of system state, $C_{1}=1 /(2 \pi)^{n(k+1) / 2}$ is a constant, and $|A|$ is the determinant of $A$ and $\|u\|_{A}^{2}=$ $u^{T} A u$.

Moreover, assuming that the measurements $z_{1}, z_{2}, \ldots, z_{k}$ are known and unrelated to each other, the distribution of $\eta_{k}$ is Gaussian, which can be expressed as

$$
\begin{aligned}
p\left[Z_{k} \mid X_{k}, q, Q, r, R\right] & \\
& =\prod_{j=1}^{k} p\left[z_{j} \mid x_{j}, r, R\right] \\
& =\prod_{j=1}^{k} \frac{1}{(2 \pi)^{m / 2}|R|^{1 / 2}} \exp \left\{-\frac{1}{2}\left\|z_{j}-h_{j}\left(x_{j}\right)-r\right\|_{R^{-1}}^{2}\right\} \\
& =C_{2}|R|^{-k / 2} \exp \left\{-\frac{1}{2} \sum_{j=1}^{k}\left\|z_{j}-h_{j}\left(x_{j}\right)-r\right\|_{R^{-1}}^{2}\right\},
\end{aligned}
$$

where $m$ denotes the dimension of measurements and $C_{2}=$ $1 /(2 \pi)^{m k / 2}$ is a constant. 
Substituting (18) and (19) into (17) yields

$$
\begin{aligned}
J=C_{1} C_{2}\left|P_{0}\right|^{-1 / 2}|Q|^{-k / 2}|R|^{-k / 2} p[q, Q, r, R] \\
=C|Q|^{-k / 2}|R|^{-k / 2} \exp \left\{-\frac{1}{2}\left[\sum_{j=1}^{k}\left\|x_{j}-f_{j-1}\left(x_{j-1}\right)-q\right\|_{Q^{-1}}^{2}\right.\right. \\
\left.\left.+\sum_{j=1}^{k}\left\|z_{j}-h_{j}\left(x_{j}\right)-r\right\|_{R^{-1}}^{2}\right]\right\},
\end{aligned}
$$

where

$$
C=\exp \left\{-\frac{1}{2}\left\|x_{0}-\widehat{x}_{0}\right\|_{P_{0}^{-1}}^{2}\right\} C_{1} C_{2}\left|P_{0}\right|^{-1 / 2} p[q, Q, r, R]
$$

Logarithm on both sides of (20) yields

$$
\begin{aligned}
\ln J= & -\frac{k}{2} \ln |Q|-\frac{k}{2} \ln |R|-\frac{1}{2} \sum_{j=1}^{k}\left\|x_{j}-f_{j-1}\left(x_{j-1}\right)-q\right\|_{Q^{-1}}^{2} \\
& -\frac{1}{2} \sum_{j=1}^{k}\left\|z_{j}-h_{j}\left(x_{j}\right)-r\right\|_{R^{-1}}^{2}+\ln C .
\end{aligned}
$$

By the logarithmic nature, $J$ and $\ln J$ share the same extreme points. The partial derivative of $J$ can be calculated by the following equations:

$$
\begin{gathered}
\left.\frac{\partial \ln J}{\partial q}\right|_{q=\widehat{q}_{k}} ^{x_{j-1}=\widehat{x}_{j-1 / k}, x_{j}=\widehat{x}_{j / k}}=0, \\
\left.\frac{\partial \ln J}{\partial Q}\right|_{Q=\widehat{Q}_{k}} ^{x_{j-1}=\widehat{x}_{j-1 / k}, x_{j}=\widehat{x}_{j / k}}=0, \\
\left.\frac{\partial \ln J}{\partial r}\right|_{r=\widehat{r}_{k}} ^{x_{j}=\widehat{x}_{j / k}}=0, \\
\left.\frac{\partial \ln J}{\partial R}\right|_{R=\widehat{R}_{k}} ^{x_{j}=\widehat{x}_{j / k}}=0 .
\end{gathered}
$$

Then, the noise statistic estimator can be derived, which is defined by

$$
\widehat{q}_{k}=\frac{1}{k} \sum_{j=1}^{k}\left[\widehat{x}_{j / k}-f_{j-1}\left(\widehat{x}_{j-1 / k}\right)\right] \text {, }
$$

$$
\begin{gathered}
\widehat{Q}_{k}=\frac{1}{k} \sum_{j=1}^{k}\left\{\left[\widehat{x}_{j / k}-f_{j-1}\left(\widehat{x}_{j-1 / k}\right)-q\right]\right. \\
\left.\times\left[\widehat{x}_{j / k}-f_{j-1}\left(\widehat{x}_{j-1 / k}\right)-q\right]^{T}\right\} \\
\widehat{r}_{k}=\frac{1}{k} \sum_{j=1}^{k}\left[z_{j}-h_{j}\left(\widehat{x}_{j / k}\right)\right] \\
\widehat{R}_{k}=\frac{1}{k} \sum_{j=1}^{k}\left\{\left[z_{j}-h_{j}\left(\widehat{x}_{j / k}\right)-r\right]\left[z_{j}-h_{j}\left(\widehat{x}_{j / k}\right)-r\right]^{T}\right\} .
\end{gathered}
$$

In (24) to (27), the smoothed estimates $\widehat{x}_{j-1 / k}$ and $\widehat{x}_{j / k}$ can be replaced by the filtered estimate $\widehat{x}_{j / j}$ or the predicted state $\widehat{x}_{j / j-1}$ as approximating solutions.

\section{Noise Statistic Estimator for UKF}

From the consideration for the nonlinear purposes, the noise statistic estimator derived above should be modified. In the linear applications, the term of $f_{j-1}\left(x_{j-1}\right)$ can be obtained by propagating each estimate $\widehat{x}_{j-1}$ through system model and $h_{j}\left(x_{j}\right)$ by propagating $\hat{x}_{j}$ through measurement equation. However, for the nonlinear field, the scaled sigma points are inserted instead of the estimate. The predicted term for UKF could be expressed as a combination of all sigma points:

$$
\left.f_{j-1}\left(x_{j-1}\right)\right|_{x_{j-1} \leftarrow \widehat{x}_{j-1 / j-1}}=\sum_{i=0}^{L} W_{i}^{m} f_{j-1}\left(\xi_{i, j-1 / j-1}\right),
$$

where $f_{j-1}\left(x_{j-1}\right)$ is approximated by UT with a precision close to that using three-order Taylor series expansion method [14].

Similarly, $h_{j}\left(x_{j}\right)$ can be calculated by

$$
\left.h_{j}\left(x_{j}\right)\right|_{x_{j} \leftarrow \hat{x}_{j / j-1}}=\sum_{i=0}^{L} W_{i}^{m} h_{j}\left(\xi_{i, j / j-1}\right) .
$$

Submitting (28) into (24) to (27) yields the noise statistic estimator for UKF:

$$
\begin{gathered}
\widehat{q}_{k}=\frac{1}{k} \sum_{j=1}^{k}\left[\widehat{x}_{j / j}-\sum_{i=0}^{L} W_{i}^{m} f_{j-1}\left(\xi_{i, j-1 / j-1}\right)\right], \\
\widehat{Q}_{k}=\frac{1}{k} \sum_{j=1}^{k}\left\{\left[\widehat{x}_{j / j}-\widehat{x}_{j / j-1}\right]\left[\widehat{x}_{j / j}-\widehat{x}_{j / j-1}\right]^{T}\right\}, \\
\widehat{r}_{k}=\frac{1}{k} \sum_{j=1}^{k}\left[z_{j}-\sum_{i=0}^{L} W_{i}^{m} h_{j}\left(\xi_{i, j / j-1}\right)\right], \\
\widehat{R}_{k}=\frac{1}{k} \sum_{j=1}^{k}\left\{\left[z_{j}-\widehat{z}_{j / j-1}\right]\left[z_{j}-\widehat{z}_{j / j-1}\right]^{T}\right\} .
\end{gathered}
$$

The unbiased properties of the noise estimates for UKF are proved in the appendix. 


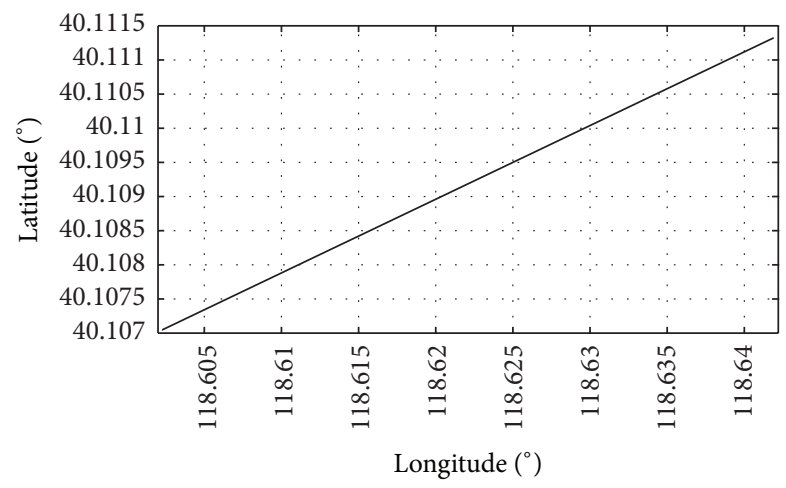

FIGURE 1: The track of land vehicle.

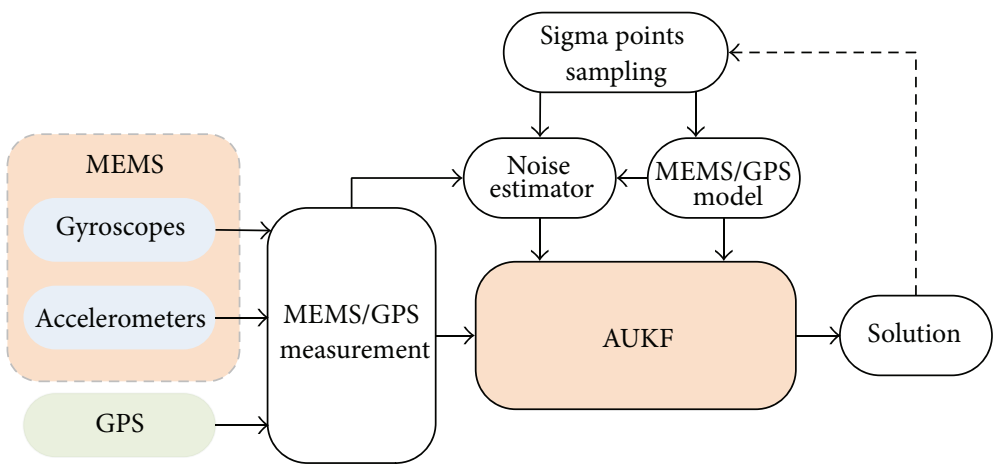

FIGURE 2: The architecture of MEMS/GPS integrated system with AUKF.

\section{Recursive Equations of Adaptive Unscented Kalman Filtering Algorithm}

Based on the UKF and its noise statistic estimator, the prediction and update steps of AUKF algorithm are as follows.

(1) Prediction. Propagating the sigma points $\xi_{i, k / k-1}$ through nonlinear state function $f_{k}(\cdot)$ yields

$$
\gamma_{i, k / k-1}=f_{k-1}\left(\xi_{i, k / k-1}\right), \quad i=0,1, \ldots, 2 n
$$

Then, according to (30) and (31), estimate the process noise $\widehat{q}_{k}$ and covariance $\widehat{Q}_{k}$, respectively.

With updated process noise parameters, compute the predicted state $\widehat{x}_{k / k-1}$ and the predicted covariance $P_{k / k-1}$ as

$$
\begin{gathered}
\widehat{x}_{k / k-1}=\sum_{i=0}^{L} W_{i}^{m} \gamma_{i, k / k-1}=\sum_{i=0}^{L} W_{i}^{m} f_{k-1}\left(\varepsilon_{i, k-1 / k-1}\right)+\widehat{q}_{k-1}, \\
P_{k / k-1}=\sum_{i=0}^{L} W_{i}^{m}\left(\gamma_{i, k-1 / k-1}-\widehat{x}_{k / k-1}\right) \\
\times\left(\gamma_{i, k-1 / k-1}-\widehat{x}_{k / k-1}\right)^{T}+\widehat{Q}_{k-1} .
\end{gathered}
$$

(2) Updating. Propagating the sigma points $\xi_{i, k / k-1}$ through nonlinear state function $h_{k}(\cdot)$ yields

$$
x_{i, k / k-1}=h_{k}\left(\xi_{i, k / k-1}\right), \quad i=0,1, \ldots, 2 n \text {. }
$$

According to (32) and (33), estimate the measurement noise $\widehat{r}_{k}$ and its covariance $\widehat{R}_{k}$, respectively.

With real-time measurement noise parameters, compute the predicted measurement $\widehat{z}_{k / k-1}$ and the covariance $P_{\widetilde{z}_{k}}$ as

$$
\begin{gathered}
\widehat{z}_{k / k-1}=\sum_{i=0}^{L} W_{i}^{m} x_{i, k / k-1}=\sum_{i=0}^{L} W_{i}^{m} h_{k}\left(\varepsilon_{i, k / k-1}\right)+\widehat{r}_{k}, \\
P_{\widetilde{z}_{k}}=\sum_{i=0}^{L} W_{i}^{m}\left(\chi_{i, k / k-1}-\widehat{z}_{k / k-1}\right)\left(\chi_{i, k / k-1}-\widehat{z}_{k / k-1}\right)^{T}+\widehat{R}_{k} .
\end{gathered}
$$

Estimate the cross-covariance $P_{\widetilde{x}_{k} \tilde{z}_{k}}$ as

$$
P_{\tilde{x}_{k} \tilde{z}_{k}}=\sum_{i=0}^{L} W_{i}^{c}\left(\gamma_{i, k / k-1}-\widehat{x}_{k / k-1}\right)\left(\chi_{i, k / k-1}-\widehat{z}_{k / k-1}\right)^{T} .
$$




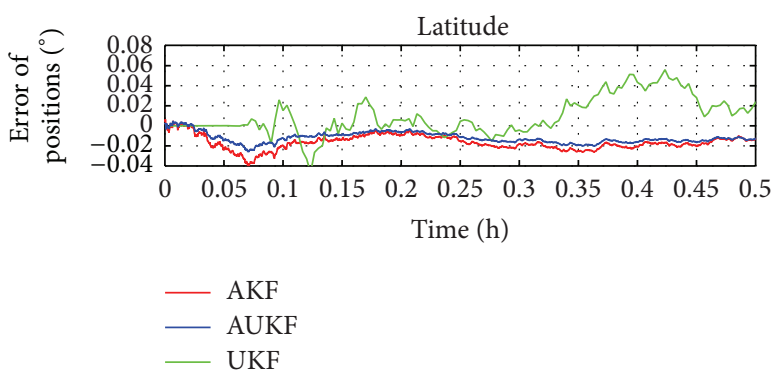

(a)

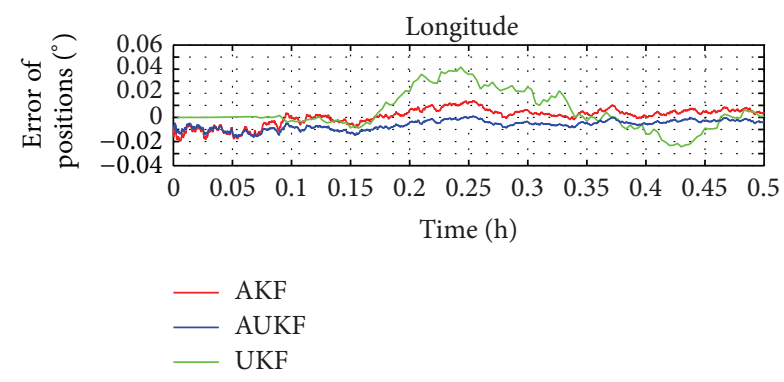

(b)

FIGURE 3: Comparison of position errors.

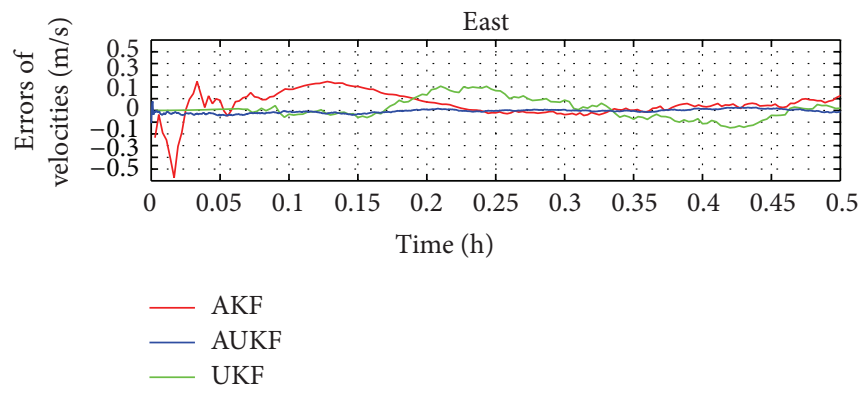

(a)

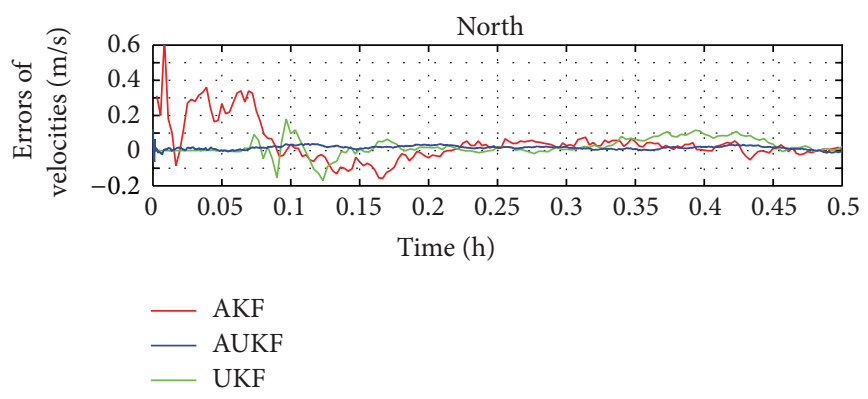

(b)

FIgURE 4: Comparison of velocity errors.

Then compute the filter gain $K_{k}$, the estimated state vector $\widehat{x}_{k / k}$, and its covariance $P_{k / k}$ :

$$
\begin{gathered}
K_{k}=P_{\tilde{x}_{k} \tilde{z}_{k}}\left(P_{\tilde{z}_{k}}\right)^{-1}, \\
\widehat{x}_{k / k}=\widehat{x}_{k / k-1}+K_{k}\left(z_{k}-\widehat{z}_{k / k-1}\right), \\
P_{k / k}=P_{k / k-1}-K_{k} P_{\widetilde{z}_{k}}\left(K_{k}\right)^{T} .
\end{gathered}
$$

\section{MEMS/GPS Integrated Navigation for Land-Vehicle Using AUKF}

Because of the highly nonlinear characteristic of MEMS/GPS, the conventional AKF based on small angle approximations is limited. Meanwhile, due to the time-varying noise stochastic properties for land-vehicle, the standard UKF in Section 1 cannot be directly applied to integrated navigation. On the other side, the modified AUKF based on a statistic estimator in Section 4 appears appropriate for the MEMS/GPS integrated navigation. Simulations are conducted to compare the performances of AKF, UKF, and AUKF.

In the simulation, the parameters of sensor errors are shown in Table 1. The initial position of vehicle is east longitude $126^{\circ}$ and north latitude $45^{\circ}$.

Figure 1 shows the trajectory of land-vehicle motion. The solid line in this figure illustrates the real simulated trajectory.

The architecture of MEMS/GPS integrated navigation with AUKF is shown in Figure 2.

As shown in Figures 3, 4, and 5, with the comparisons with AKF and UKF, the AUKF with noise estimator could obviously improve the accuracy of the velocity solutions. In addition, as shown in Figure 3, because the noise statistic estimators are designed in AKF and AUKF, the position errors of the two filters exhibit similar characteristics at most of the time in this simulation, and the performance of AUKF is slightly better. However, which is also seen in Figure 3, there 


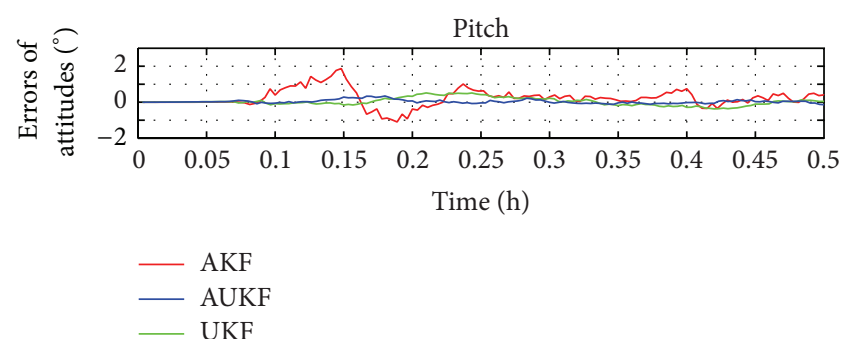

(a)

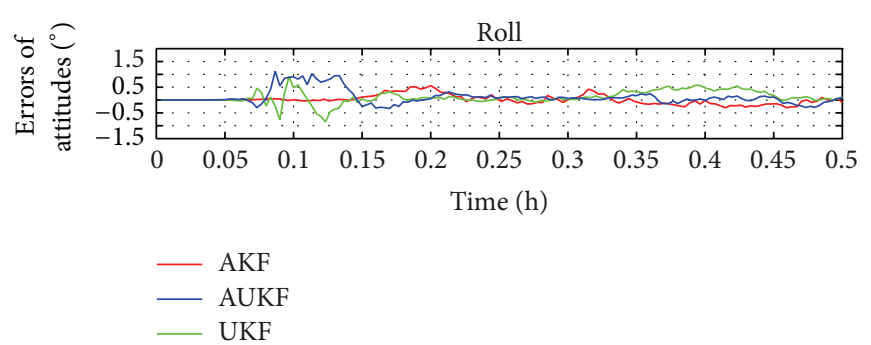

(b)

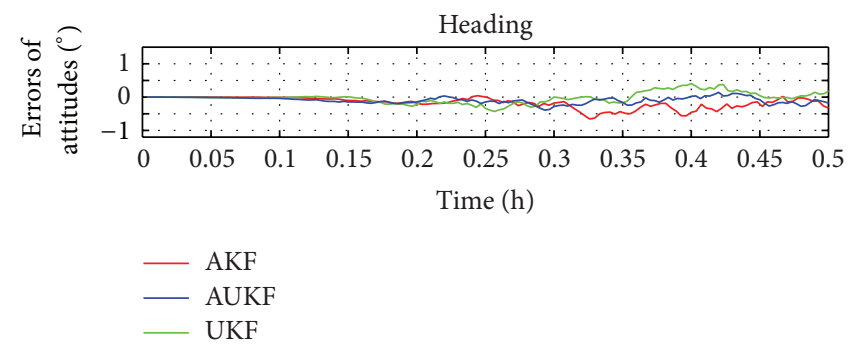

(c)

FIGURE 5: Comparison of attitude errors.

TABLE 1: Parameters of sensor errors.

\begin{tabular}{lcc}
\hline Sensor & Characteristic & Value \\
\hline MEMS Gyro & Drift & $5^{\circ} / \mathrm{h}$ \\
MEMS Gyro & Measuring white noise & $0.5^{\circ} / \mathrm{h}$ \\
MEMS accelerometer & Bias & $2 \mathrm{mg}$ \\
MEMS accelerometer & Measuring white noise & $50 \mu \mathrm{g}$ \\
GPS (position) & Measuring white noise & $6 \mathrm{~m}$ \\
GPS (velocity) & Measuring white noise & $0.01 \mathrm{~m} / \mathrm{s}$ \\
\hline
\end{tabular}

are so notable vibrations for UKF in which stable estimate errors of positions could not be provided, which are mainly caused by the quickly changed system noises.

Figure 4 shows that the AUKF always has smaller velocity errors than AKF and UKF. And Figure 5 shows that at most of the time the AUKF scheme has a better performance on attitudes errors. The AKF and UKF solutions have large vibration errors in both Figures 4 and 5 . That is because the velocity errors are related to the attitude errors, especially to the pitch and roll errors of AKF and UKF. Because of the strong nonlinear properties of system, it is difficult that AKF cannot be effectively operated for navigation. Meanwhile, for UKF solutions, the curves of speed errors are extremely similar to those of horizontal attitude errors.

The simulation results indicate that if the AUKF scheme is considered the filtering solution, there are only small variations impacting on the performance of MEMS/GPS integrated navigation system, and this system has an excellent robustness. However, long processing time would cause slight divergence of the attitude errors, sequentially the velocity and position errors.

\section{Conclusions}

This study has developed an AUKF approach to improve the navigation performance of MEMS/GPS integrated system for land-vehicle applications. By treating this problem within conventional UKF framework, the noise estimator is adopted and could effectively estimate the process and measurement noise characteristics online. The results indicate that the proposed AUKF algorithm could efficiently improve the navigation performance of land-vehicle integrated navigation system. By comparing with AKF and UKF methods, the AUKF solution has a more stable and superior performance.

\section{Appendix}

The process noise and measurement noise statistic properties are computed by estimator in (30) to (33). Their unbiased properties are proved as follows.

According to (8), the predicted state at time step $j$ is given as

$$
\widehat{x}_{j / j-1}=\sum_{i=0}^{L} W_{i}^{m} f_{j-1}\left(\xi_{i, j-1 / j-1}\right)+q .
$$

Hence the mean of the process noise can be expressed as

$$
\begin{aligned}
E\left[\widehat{q}_{k}\right] & =\frac{1}{k} \sum_{j=1}^{k}\left[\widehat{x}_{j / j}-\sum_{i=0}^{L} W_{i}^{m} f_{j-1}\left(\xi_{i, j-1 / j-1}\right)\right] \\
& =\frac{1}{k} \sum_{j=1}^{k}\left[\widehat{x}_{j / j}-\widehat{x}_{j / j-1}+q\right] .
\end{aligned}
$$


From (15), we have

$$
\widehat{x}_{j / j}=\widehat{x}_{j / j-1}+K_{j}\left(z_{j}-\widehat{z}_{j / j-1}\right) .
$$

Substituting (A.3) into (A.2) yields

$$
\begin{aligned}
E\left[\widehat{q}_{k}\right] & =\frac{1}{k} \sum_{j=1}^{k}\left[\widehat{x}_{j / j}-\widehat{x}_{j / j-1}+q\right] \\
& =\frac{1}{k} \sum_{j=1}^{k}\left[K_{j}\left(z_{j}-\widehat{z}_{j / j-1}\right)+q\right] .
\end{aligned}
$$

When the posteriori mean and covariance are known, the output residual vector of UKF is zero-mean Gaussian white noise and we see

$$
E\left[z_{j}-\widehat{z}_{j / j-1}\right]=0
$$

From (A.4) and (A.5), the mean of the process noise is

$$
E\left[\widehat{q}_{k}\right]=\frac{1}{k} \sum_{j=1}^{k} E\left[\left(z_{j}-\widehat{z}_{j / j-1}\right)+q\right]=q .
$$

Thus, the estimate of the process noise noted by (24) is unbiased.

Similarly, the estimate of the measurement noise is

$$
E\left[\widehat{r}_{k}\right]=r .
$$

The unbiased properties for the estimates of noises are proved.

\section{Conflict of Interests}

The authors declare that they have no conflict of interests regarding the publication of this paper.

\section{Acknowledgments}

Funding for this work was provided by the National Nature Science Foundation of China under Grant nos. 61374007 and 61104036. The authors would like to thank all the editors and anonymous reviewers for improving this paper.

\section{References}

[1] S. Mizukami, M. Sugiura, Y. Muramatsu, and H. Kumagai, "MEMS GPS/INS for micro air vehicle application," in Proceedings of the 17th International Technical Meeting of the Satellite Division of the Institute of Navigation (ION GNSS '04), pp. 819824, September 2004.

[2] X. Niu, S. Nassar, and N. El-Sheimy, "An accurate land-vehicle MEMS IMU/GPS navigation system using 3D auxiliary velocity updates," Navigation, Journal of the Institute of Navigation, vol. 54, no. 3, pp. 177-188, 2007.

[3] S. Y. Cho and B. D. Kim, "Adaptive IIR/FIR fusion filter and its application to the INS/GPS integrated system," Automatica, vol. 44, no. 8, pp. 2040-2047, 2008.
[4] S. Y. Cho and W. S. Choi, "Robust positioning technique in low-cost DR/GPS for land navigation," IEEE Transactions on Instrumentation and Measurement, vol. 55, no. 4, pp. 1132-1142, 2006.

[5] A. Gelb, Applied Optimal Estimation, The MIT press, 1974.

[6] C. Hide, T. Moore, and M. Smith, "Adaptive Kalman filtering for low-cost INS/GPS," Journal of Navigation, vol. 56, no. 1, pp. 143-152, 2003.

[7] C. W. Hu, Congwei, W. Chen, Y. Chen, and D. Liu, "Adaptive Kalman filtering for vehicle navigation," Journal of Global Positioning Systems, vol. 2, no. 1, pp. 42-47, 2003.

[8] A. H. Mohamed and K. P. Schwarz, "Adaptive Kalman filtering for INS/GPS," Journal of Geodesy, vol. 73, no. 4, pp. 193-203, 1999.

[9] D. Simon, Optimal State Estimation: Kalman, H Infinity, and Nonlinear Approaches, Wiley, 2006.

[10] S. J. Julier and K. U. Jeffrey, "A general method for approximating nonlinear transformations of probability distributions," Tech. Rep., Robotics Research Group, University of Oxford, Oxford, UK, 1996.

[11] Z. Jiang, Q. Song, Y. He, and J. Han, "A novel adaptive unscented kalman filter for nonlinear estimation," in Proceedings of the 46th IEEE Conference on Decision and Control (CDC '07), pp. 4293-4298, New Orleans, LA, USA, December 2007.

[12] S. J. Julier, "The scaled unscented transformation," in Proceedings of the American Control COnference, pp. 4555-4559, May 2002.

[13] S. Särkkä, “On unscented Kalman filtering for state estimation of continuous-time nonlinear systems," IEEE Transactions on Automatic Control, vol. 52, no. 9, pp. 1631-1641, 2007.

[14] L. Zhao, X.-X. Wang, H.-X. Xue, and Q.-X. Xia, "Design of unscented Kalman filter with noise statistic estimator," Control and Decision, vol. 24, no. 10, pp. 1483-1488, 2009. 


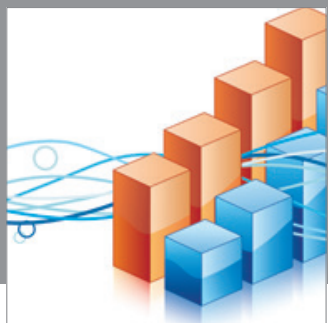

Advances in

Operations Research

mansans

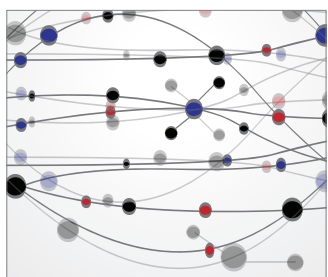

The Scientific World Journal
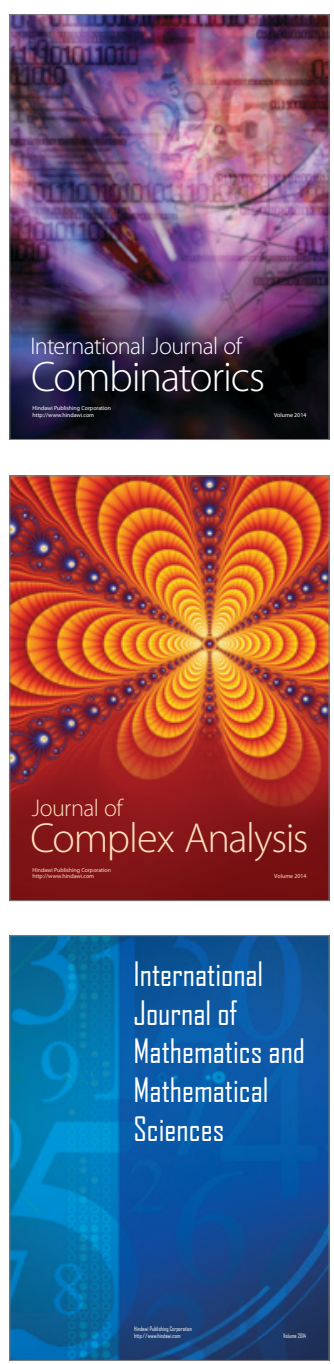
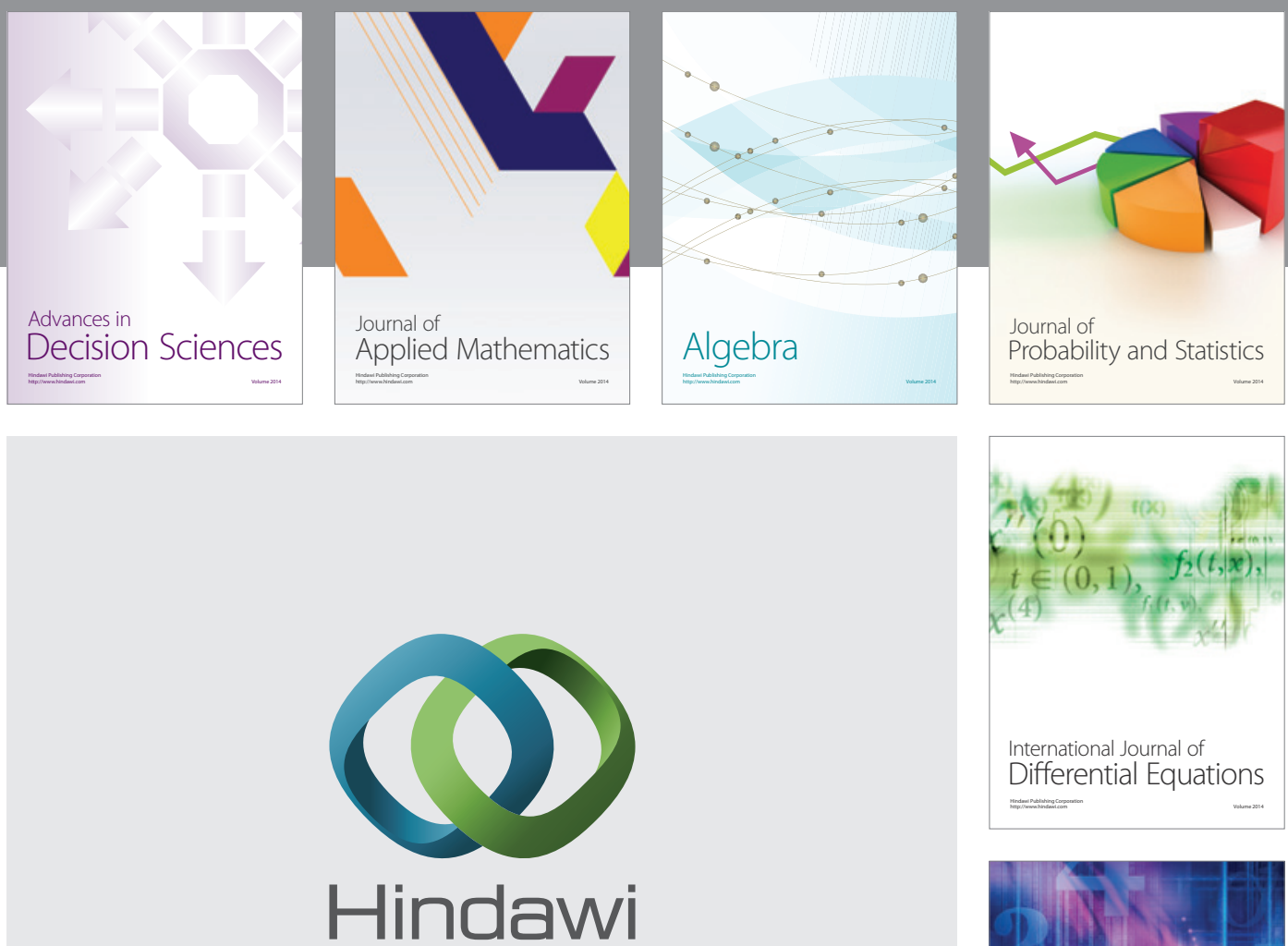

Submit your manuscripts at http://www.hindawi.com
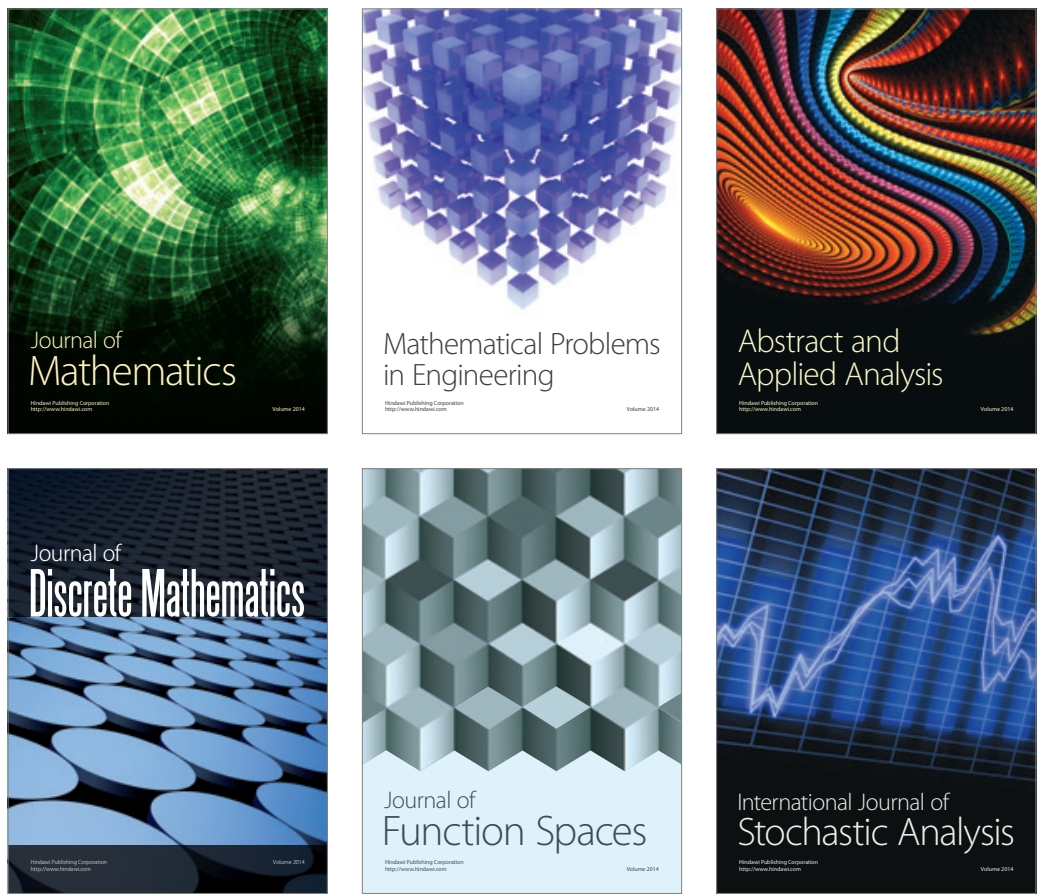

Journal of

Function Spaces

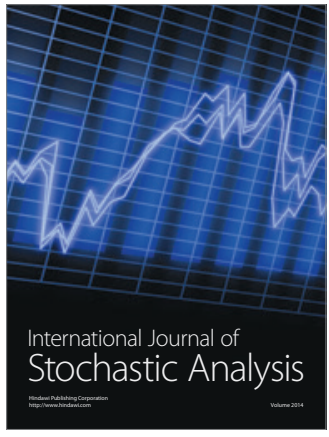

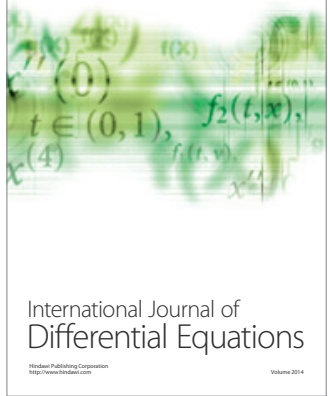
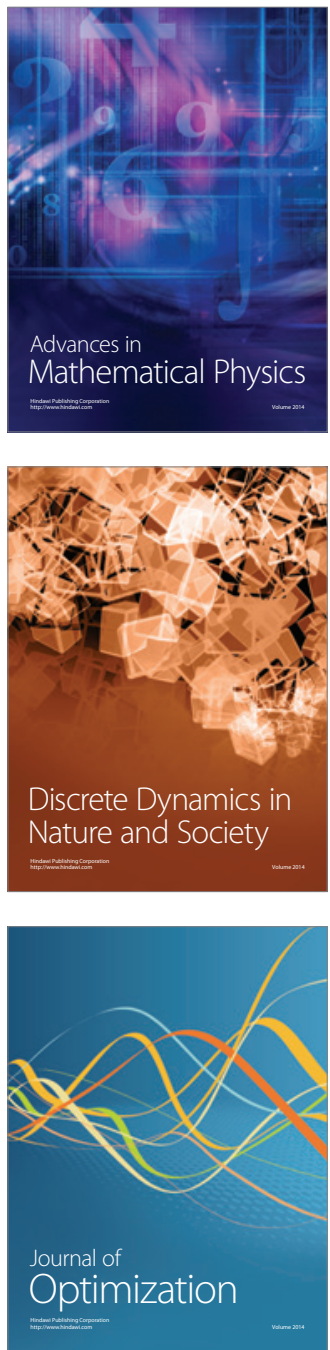